\title{
Performance Assessment Of Wireless Power Transfer Links For Implantable Microsystems
}

\author{
H. Dinis*, P. M. Mendes, Senior Member, IEEE \\ Departamento de Eletrónica Industrial \\ Universidade do Minho \\ Guimarães, Portugal \\ *a61776@alunos.uminho.pt
}

\begin{abstract}
Wireless power transfer is a hot topic due to the growth of implanted device solutions which, as they get smaller and smarter, demand new solutions to power them up. These solutions need to keep the power level, namely SAR (specific absorption ratio) below a determined safety standard and to allow the device to be as small as possible. Current fabrication techniques allow the creation of ultra-small 3D antennas integrated on silicon wafers, which can lead to the miniaturization of implantable devices due to the possible reduction or even elimination of battery size. To evaluate this possibility, the antenna must be tested in conditions close to real working conditions, requiring the use of human body phantoms. This paper proposes a solution to measure the power received by an ultra-small antenna placed inside a phantom, without the use of coaxial cables attached to the device. Instead, an optoelectronic mechanism is used to route the received power to an optical fiber, and an optical spectrum analyzer is used to measure the received power..
\end{abstract}

Keywords-Implantable device, Wireless power transfer, Human body phantom, Antenna characterization.

\section{INTRODUCTION}

The growth of smaller and smaller implanted device solutions demands for new solutions to power them up [1], since the available room is very small and the power required by new features is increasing. Usually, batteries are used to power the devices, but since their energy storage is limited and size dependent, it can limit the lifetime of the device or require periodic replacement. Due to this sizable restriction, wireless power transfer (WPT) has been a growing field of study in recent years, as it presents an unlimited power source and grants the opportunity to reduce battery size or even eliminate the need for batteries entirely, which can lead to even smaller devices and new applications. Some of the new applications are related to the biomedical field, such as implantable chemical sensors [1], glucose and oxygen sensors for diabetics [2], orthopedic implants deformation monitors [3], neural implants [4], intra-cranial pressure sensors [5] and cardioverterdefibrillators [6]. A visual representation of these devices and their power source and is shown in Fig. 1.

Solutions to power up such microdevices need to satisfy two constraints. One is to keep the power level, namely
SAR (specific absorption ratio, defined as the rate at which electromagnetic energy is absorbed by a lossy object) below a pre-defined safety standard, and the other is to keep the power sub-system (antenna and energy storage) as small as possible. This way, optimization of WPT links is required to allow the maximum power delivery to the smallest microsystem possible. Radiofrequency (RF) electromagnetic radiation is a promising energy source, since it's suitable for long distance energy transportation and it's available almost everywhere. Ultimately, resorting to ambient energy sources - energy harvesting - could provide power indefinitely, being an ideal method of device powering. The fact that research in this field has significantly grown demonstrates that this approach is feasible and research worthy [7].

Current fabrication techniques allow the creation of ultrasmall 3D devices, namely ultra-small antennas for human body implantation [8]. Due to their small size, it was necessary to develop a fabrication methodology to fabricate the antenna on top of a silicon wafer containing transmission lines and various electronic components. Such antennas have been fabricated and are now being tested for different scenarios [9]. An HFSS model of the antenna is presented in Fig. 2. Since such devices should be implantable, performance tests should be performed under working conditions as similar as possible to normal operating conditions found inside the implanted body. This requires performance assessment to be made using liquid phantoms that mimic the human body's electrical properties at desired frequencies, similar to what was used in simulation.

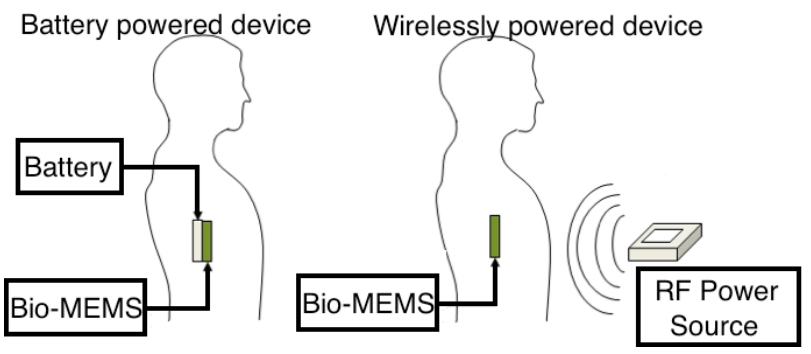

Fig. 1. Representation of conventional implantable and wirelessly powered devices. Adapted from [9]. 

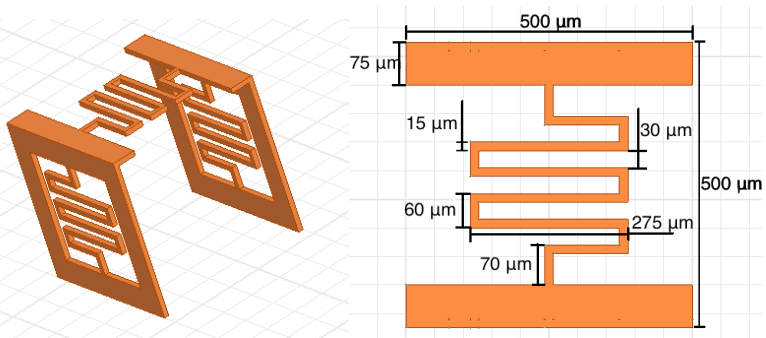

Fig. 2. HFSS model of the ultra-small antenna (left) and its' dimensions from a top view (right).

In this paper we present solutions to obtain materials with the desired dielectric properties for the required biological tissues. We also propose an experimental setup to enable the characterization of such small antennas. Since the antenna is physically very small, when using the standard measurement setup, the interface to the VNA port is much larger then the antenna itself. In that case is difficult to determine how much power the antenna is collecting and how much power could be being collected by the interface structure.

\section{HUMAN BODY PHANTOMS}

The human body interferes with antenna characteristics, such as input impedance, radiation directivity and radiation efficiency. In order to account for those effects in simulations and experiments, we can use body phantoms, which are biological tissue-equivalent materials. They mimic electrical properties of biological tissues over, such as permittivity and conductivity, over a frequency range and can be either solid or liquid, allowing them to take any shape desired for a given purpose [14]

\section{A. Dielectric Properties of Biological Tissues}

The dielectric characteristics of a substance or material are key in determining how it interacts with an applied electromagnetic field. Of these properties, and for the required purposes, the most important are electrical permittivity and conductivity.

Propagation of an RF wave through a non-magnetic material can be described with just the complex permittivity of said material. The complex absolute permittivity is given by the following formula.

$$
\hat{\varepsilon}(\omega)=\varepsilon^{\prime}+j \varepsilon^{\prime \prime}=\varepsilon^{\prime}+\frac{\sigma}{j \omega}
$$

A complex relative permittivity may also be defined, where $\omega \mathrm{rad} / \mathrm{s}$ is the angular frequency and $\varepsilon_{0}=8.854 \times 10^{-12}$ $\mathrm{F} / \mathrm{m}$ is the permittivity of free-space.

$$
\hat{\varepsilon}_{r}(\omega)=\varepsilon_{r}(\omega)+\frac{\sigma(\omega)}{j \omega \varepsilon_{0}}
$$

According to [10], the real part of permittivity, $\varepsilon^{\prime}$, is a measure of how much energy from an external electric field is stored in a material. The imaginary part of permittivity, $\varepsilon^{\prime \prime}$, is called the loss factor, and is a measure of how dissipative a material is to an external electric field. Also, the real part of the relative permittivity, $\varepsilon_{\mathrm{r}}$, and the conductivity $\sigma(\mathrm{S} / \mathrm{m})$, are all that is required to describe lossy, frequency-dependent media, assuming non-magnetic materials.

Gabriel et al $[11,12,13]$ have studied and determined the dielectric properties of several biological tissues, some of which are shown in Fig. 3. It is possible to see that the values are changing along with the frequency. In general, the real part of permittivity decreases with increasing frequency, as opposed to conductivity, that increases.

\section{B. Phantom Development}

Although solid phantoms don't require an outer shell to contain them, which could introduce some uncertainty in the measurements, we have opted to use liquid phantoms. This
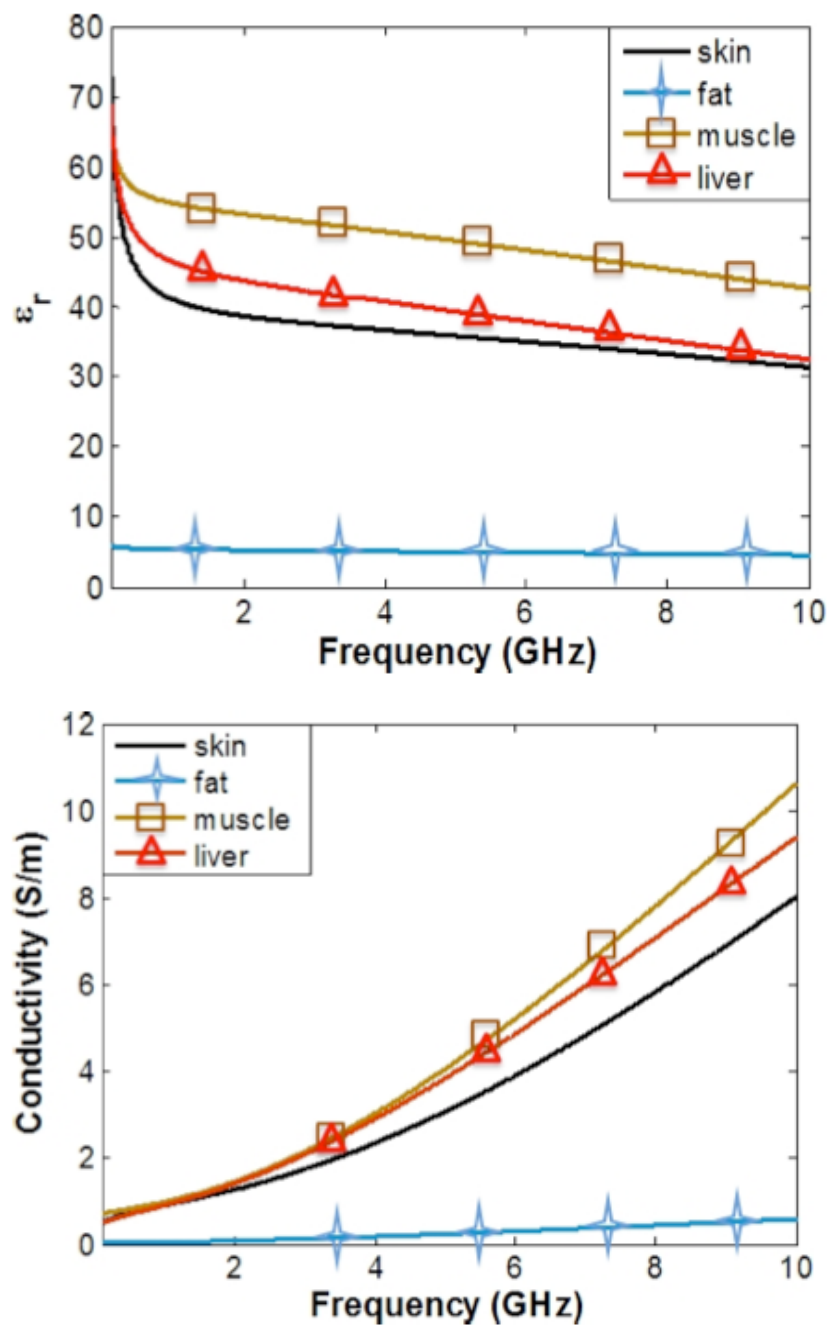

Fig. 3. Relative permittivity $\varepsilon_{\mathrm{r}}$ (top) and conductivity $\sigma$ (bottom) for skin, fat, muscle and liver tissues over a frequency range. [10]. 
choice is by virtue of our need to dip the antenna and electronic circuitry inside of it, and also the sizable easiness of production and control of phantom properties, unlikely to solid ones.

The Federal Communications Commission (FCC), on the topic of evaluation of the specific absorption rate (SAR) associated with mobile phone usage [15], suggests values for the dielectric constant and conductivity of human head and body tissue at several frequencies, as well as some recipes of solutions that mimic these properties.

As to this point, we haven't started to use FCC's suggested recipes, having decided to firstly perform some tests with less complex solutions, namely water - ethyl alcohol solutions, in order to evaluate the feasibility of tissue mimicking solutions. The graphics presented in Fig. 4 contain the measurement of relative permittivity and conductivity of two solutions. The first one consists purely of
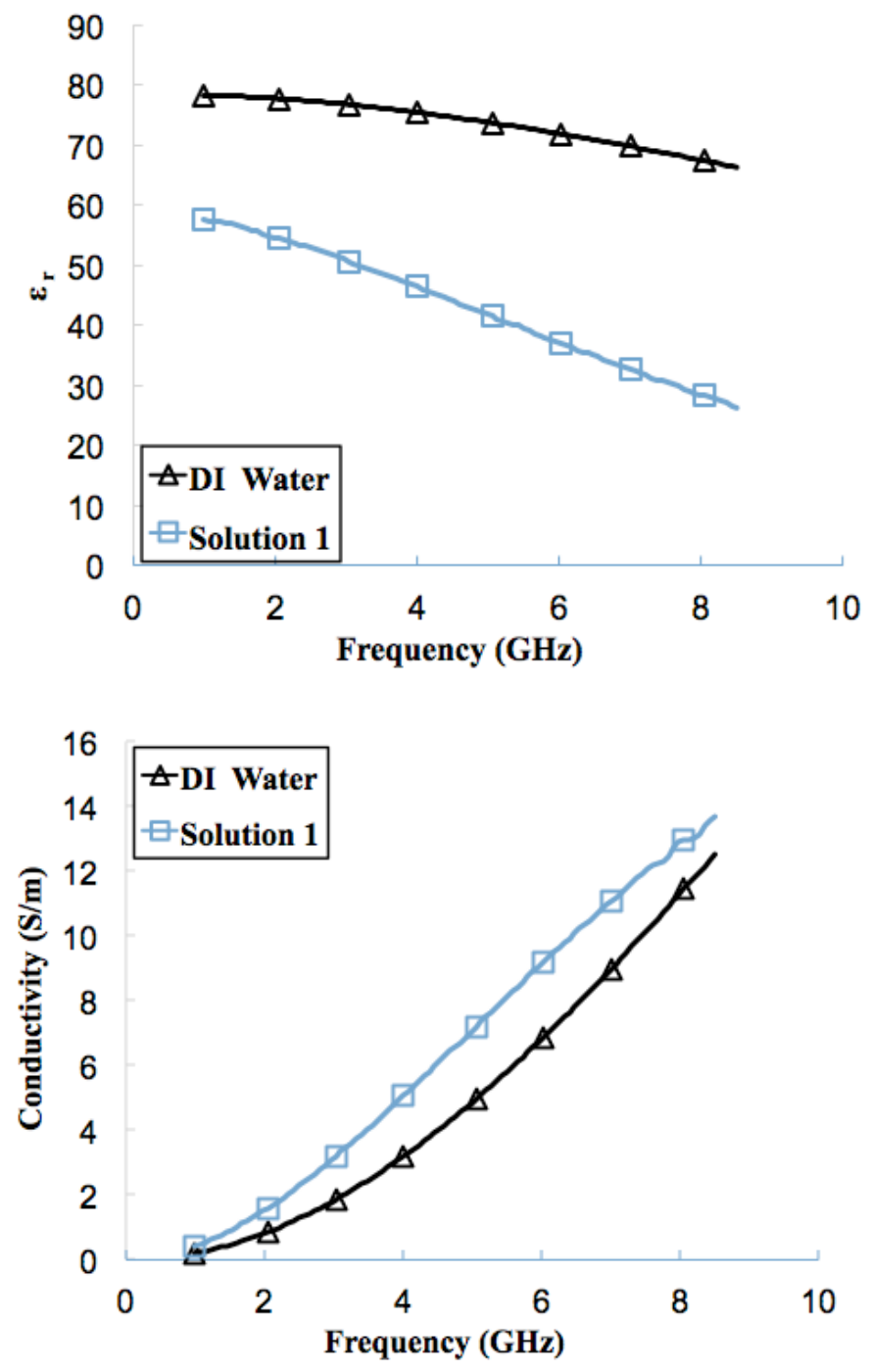

Fig. 4. Relative permittivity $\varepsilon_{\mathrm{r}}$ (top) and conductivity $\sigma$ (bottom) for DI water and a $53.3 \% \mathrm{v} / \mathrm{v}$ DI water and $46.7 \% \mathrm{v} / \mathrm{v}$ ethyl alcohol solution. deionized (DI) water, and serves as a reference line to the second solution, named solution 1 , which consists of $53.3 \mathrm{v} / \mathrm{v} \%$ DI water and $46.7 \mathrm{v} / \mathrm{v} \%$ ethyl alcohol. As we can observe, the relative permittivity of DI water alone is very high and unsuited to mimic biological tissue on its own. Adding ethyl alcohol, however, a substance with much smaller permittivity will cause solution 1's permittivity to drop its value. After some iterations, it was possible to determine the values shown above for the composition of solution 1 are adequate to obtain a permittivity value very close to that of human body tissue at $2.45 \mathrm{GHz}$, stated by the FCC [15] to be 52.7 at that same frequency. Solution 1 also presents a value of conductivity around 2 , as suggested by the FCC and confirmed by Gabriel et al (Fig. 3) for the selected frequency. Although it seems to be possible to produce satisfactory phantoms with only these two ingredients, it was noted that using ethyl alcohol results in unstable solutions, as alcohol easily evaporates, thus changing the solution's content over time, not allowing for repetitive measurements over a considerable period of time, arising the need for better and more stable solutions, such as the ones suggested in [15, 17-19].

\section{SMALL-ANTENNA CHARACTERIZATION SETUP}

As it was presented previously [9, 16], any experiment with antenna's of such small dimensions proves to be a challenge, simply because the mounting structure of the antenna is usually a couple orders of magnitude than the antenna itself. This fact can lead to the suspicion that any radiation produced by the device is mostly due to the structure and not the antenna itself.

In order to eliminate said suspicion, a fabrication process was devised where the antenna self-folds into position on top of a silicon wafer containing transmission lines, pads and electronic components $[8,9]$. With this method, we will be able to test the antenna with a very small mounting structure, thus allowing us to more easily reach an irrefutable conclusion about the antenna's compliance with the expected performance. However, such methodology still does not eliminate a potential source of power reception. The proposed solution is based on the setup shown in Fig. 5.

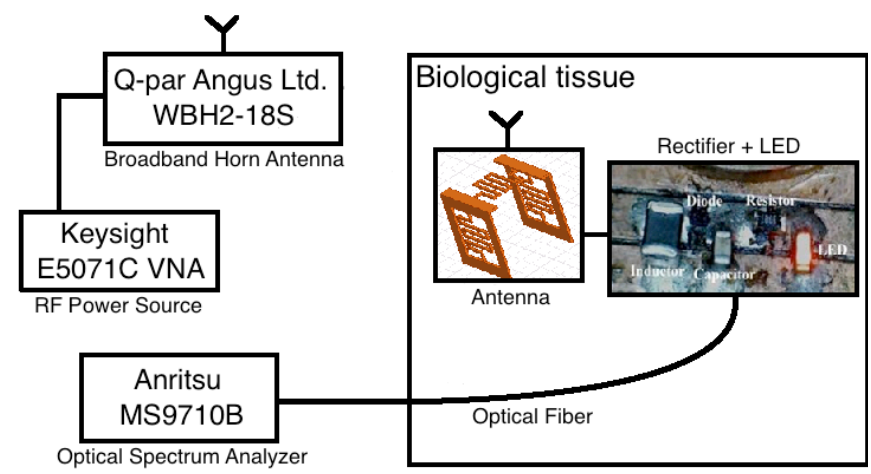

Fig. 5. Block representation of the proposed experimental setup for antenna performance assessment. 
In a standard setup, we have two options. One consists of interfacing the antenna to a board that will interface to an RF cable. Such solutions suffer from the problems discussed in [9]. The other solution is based on the fact that it is possible to fabricate a very small device containing the antenna, a capacitor, and a charging circuitry. This device would be surrounded in a human body phantom, in order to simulate real working conditions, and an external RF power source would radiate into de phantom, where we would expect the antenna to receive some of the power, and charge the capacitor. Then it would be possible to measure the received power, at the DC level. Even though this approach sounds viable, it is necessary to bare in mind that due to the antenna's reduced dimensions and efficiency, we can't connect wires to measure the antenna's power output, because, again, wires themselves can act as antennas and influence the system's performance [16].

As a workaround to this problem, we suggest an indirect, yet viable and reliable method to verify the antenna's performance. Instead of the capacitor and its' charging circuitry, an LED and an AC/DC rectifier would be fabricated together with the antenna. The procedure would then be the same, yet the power received will be routed outside the phantom, through an optical fiber, and the power received will be measured with an optical spectrum analyzer, as proposed by Fig. 5 .

Finally, experimentation in real tissue could be possible, since we only need to insert a small optical fiber to route the power outside the body under test.

\section{CONCLUSIONS}

Implantable devices have recently been decreasing in size, thanks to advances in fabrication and integration technologies. These technologies allow the fabrication of antennas on top of silicon wafers that, in turn, can lead to smaller, wirelessly powered microdevices. Even though testing this possibility in real biological tissues poses an obstacle, it was possible to conclude in this paper that human body phantoms present a very good alternative to real tissues, replicating their most significant electrical properties. We also propose an experimental setup that will allow us to verify the antenna's power supplying capabilities, which will be a very important step towards the characterization of such tiny devices.

\section{ACKNOWLEDGMENT}

This work was supported by Portuguese Foundation for Science and Technology: FCT-PTDC/EEI-TEL/2881/2012, Programa Operacional Temático Fatores de Competitividade (COMPETE) and Fundo Comunitário Europeu FEDER.

\section{REFERENCES}

[1] M. C. Frost and M. E. Meyerhoff, "Implantable chemical sensors for real-time clinical monitoring: progress and challenges," Curr. Opin. Chem. Biol., vol. 6, no. 5, pp. 633-641, 2002.

[2] B. D. McKean and D. A. Gough, A telemetry-instrumentation system for chronically implanted glucose and oxygen sensors, IEEE Trans. Biomed. Eng., vol. BME-35, 1988, pp. 526532.

[3] K. Van Schuylenbergh, R. Puers, F. Rodes, F. Burny,M Donkerwolcke, F. Moulart,"Monitoring Orthopaedic Implants Using Active Telemetry," Engineering in Med and Biol Soc, vol. 6, 1992,pp. 2672-2673.

[4] K. Wise, D. Anderson, J. F. Hetke, D. Kipke, and K. Najafi, "Wireless implantable microsystems: high-density electronic interfaces to the nervous system," Proc. IEEE, vol. 92, no. 1, pp. 7697, Jan. 2004

[5] M. Wenzel," Implantable telemetric pressure sensor system for longterm monitoring of therapeutic implants," Healthy Aims Dissemination Day http://www.healthyaims.org, 2004.

[6] M. Rasouli and L. S. J. Phee, "Energy sources and their development for application in medical devices," Expert Rev. Med. Devices, vol. 7 , no. 5, pp. 693-709, 2010

[7] P. Mitcheson, E. Yeatman, G. K. Rao, A. S. Holmes, and T. C. Green, "Energy harvesting from human and machine motion for wireless electronic devices," Proc. IEEE, vol. 96, no. 9, pp. $1457-$ 1486, 2008.

[8] S. Gomes, J. Fernandes, P. Anacleto, P. M. Mendes, "Ultra-small energy harvesting microsystem for biomedical applications", Proc. $44^{\text {th }}$ European Microwave Conference, pp. 660-663, Italy, 2014.

[9] H. Dinis, P. Anacleto, J. Fernandes, P. M. Mendes, "Characterization of Chip-Size Electrically-Small Antennas for Smart Wireless Biomedical Devices", The 9th European Conference on Antennas and Propagation (EuCAP), 2015, in press.

[10] Yvanoff, Marie, "LC Sensor for biological tissue characterization" (2008). Thesis. Rochester Institute of Technology.

[11] C. Gabriel, S. Gabriel and R. Corthout, "The dielectric properties of biological tissues:I.Litterature survey,"Phys. Med. Biol., vol. 41, 1996.

[12] S. Gabriel, R.W. Lau and C. Gabriel "The dielectric properties of biological tissues:II.Measurements in the frequency range $10 \mathrm{~Hz}$ to 20GHz,'Phys. Med. Biol.,vol. 41,1996.

[13] S. Gabriel, R.W. Lau and C. Gabriel, "The dielectric properties of biological tissues:III.Parametric models for the dielectric spectrum of tissues,'Phys. Med. Biol.,vol. 41,1996.

[14] T. Onishi, S. Uebayashi. Biological Tissue-Equivalent Phantoms Usable in Broadband Frequency Range. NTT DoCoMo Technical Journal. 7, no.4, pp.61-65.

[15] FCC OET Bulletin 65 (Edition 97-01) Supplement C (Edition 01-01), "Evaluating Compliance with FCC Guidelines for Human Exposure to Radiofrequency Electromagnetic Fields", June 2001

[16] Ho J.S., Yeh A.J., Neofytou E., Kim S., Tanabe Y., Patlolla B., et al. "Wireless power transfer to deep-tissue microimplants". Proc Natl Acad Sci USA. 2014 Jun 3; 111(22):7974

[17] SPORTON INTERNATIONAL (KUNSHAN) INC, "FCC SAR Test Report", February 2013.

[18] T. Yilmaz, T. Karacolak, E. Topsakal, "Characterization of Muscle and Fat Mimicking Gels at MICS and ISM Bands $(402-405 \mathrm{MHz}$ and 2.40-2.48 GHz)".

[19] Y. Gimm, "General Method of Formulating The Human Tissue Simulant Liquid For SAR Measuremente”. EMC'04, 2004, Sendai. pp. 561-564. 